\title{
A Dual Frequency Dielectric Resonator Antenna for Wireless Communication Applications
}

\author{
N.Ananda Rao, M.Sekhar
}

\begin{abstract}
A dual frequency Dielectric Resonator antenna for wireless communication applications in the $S$ and $C$ bands with an operating frequency of $2.65 \mathrm{GHz}$ and $4.62 \mathrm{GHz}$ is presented. The patch is a dielectric material with a high dielectric constant value of 20 . A $50 \Omega$ strip line is considered as feed and is coupled to the dielectric radiator via the rectangular slot etched in ground plane. The slot etched in ground plane is made so as to facilitate the power form the feed line to the radiator. The overall dimension of the antenna is $100 \mathrm{~mm} \times 35 \mathrm{~mm} \times 0.8 \mathrm{~mm}$. A dual frequency antenna has been proposed which will be operational at the frequencies of $2.65 \mathrm{GHz}$ and $4.62 \mathrm{GHz}$ with a gain of $4.42 \mathrm{~dB}$ and $7.78 \mathrm{~dB}$ respectively. Low cost FR4 material is been used as the laminate base for the antenna which will act as the dielectric material.
\end{abstract}

Keywords- Compact, Stacked Patch, WIRELESS COMMUNICATION Applications.

\section{INTRODUCTION}

Though microstrip antennas are having considerable advantageous features when compared to conventional antennas in terms of size and power consumption still they have some limitations in terms of some antennas parameters like gain and bandwidth. This limitation is because of the physical structure of the patch antenna which is inevitable. The basic structure of microstrip antenna consists of conductors and dielectric materials. The usage of the conducting material as a source for radiation and the implementation of the above said feeding technique are the fundamental sources for the limitations of microstrip antennas.

The radiating element will be placed on the dielectric material and on the other side we will be having a ground plane. When the radiator is excited by power there will be a generation of some unwanted waves in the substrate which are called as surface waves which flow in the substrate and will reduce the efficiency of the antenna. This effect will be more in case of microstrip feed line as both the conductors are on the same plane and the generation of the unwanted surface waves will be very high.

In the case of coaxial feed the conducting pin which carry the power from transmitter to patch which surpasses from substrate. This will lead to the generation of the surface waves which will reduce the antenna efficiency.

Researchers around the world proposed different techniques to overcome these limitations and enhance gain and Bandwidth of the microstrip antennas.

In [1] the authors proposed a air gap in between the ground and substrate layers.
Revised Manuscript Received on December 15, 2019.

N.Ananda Rao, Assistant Professor, Dept. of E.C.E, Vignan's Foundation For Science Technology and Research, Guntur, India.

M.Sekhar, Assistant Professor, Dept. of E.C.E, Vignan's Foundation For Science Technology and Research, Guntur, India.
In [2] a technique has been proposed to increase the gain of the antenna by taking a group of antennas and joining them as an array such that the power from all the individual elements will combine and form a single beam. In [3] Stacked patch techniques has been proposed which can be used for both gain enhancement and bandwidth enhancement depending upon the dimensions of the parasitic patch element.

In [4] the authors proposed a air gap in between the ground and substrate layers. In [5] a technique has been proposed to increase the gain of the antenna by taking a group of antennas and joining them as an array such that the power from all the individual elements will combine and form a single beam. In [6] Stacked patch techniques has been proposed which can be used for both gain enhancement and bandwidth enhancement depending upon the dimensions of the parasitic patch element. But all these techniques have complexity in designing either in terms of designing or in terms of cost. So to overcome these limitations we proposed a technique of Dielectric Resonator Antenna(DRA) where we use Dielectric material as a radiator instead of conductor. In [7] an four element antenna array has been proposed where all the four elements are been excited with a single feed and because of this the control over the phase of the input signal is not possible which is very essential in the phased array antenna applications especially in RADRA's where we need to steer the beam in different directions. In [8] an four element antenna array has been proposed where a separate feeding structure has been used to excite the individual antenna elements. For this proximity feeding concepts are been used which will require two different substrates for the development of the antenna and is not encouraged as it will increase the cost of the antenna. In [9] an four element antenna array has been proposed to achieve high gain but to excite the antenna elements a complex directional coupler network has been used and this is bringing difficulty in antenna fabrication and also the feed network is generating some surface waves which will reduce the efficiency of antenna.

In this paper, A dual frequency DRA patch antenna has been designed for the wireless communication applications with operating frequency of $2.65 \mathrm{GHz}$ and $4.62 \mathrm{GHz}$. Microstrip line is used for excitation. For the required operating frequency the ground plane is etched with a slot right below the DRA.

\section{PROPOSED ANTENNA DESIGN}

A dual frequency DRA which operates at $2.65 \mathrm{GHz}$ and $4.62 \mathrm{GHz}$ is proposed. Low cost flame retardent material has been used as the substrate material with a thickness of $0.8 \mathrm{~mm}$.

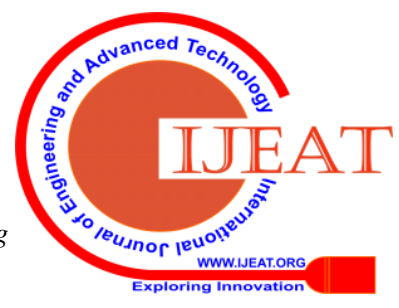




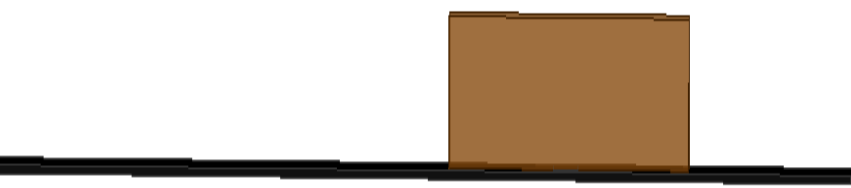

(a) Side View
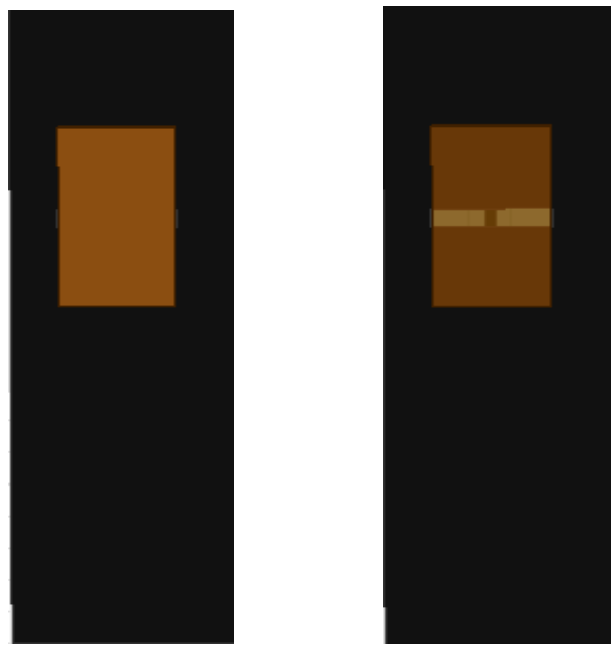

(b) Top View of proposed antenna

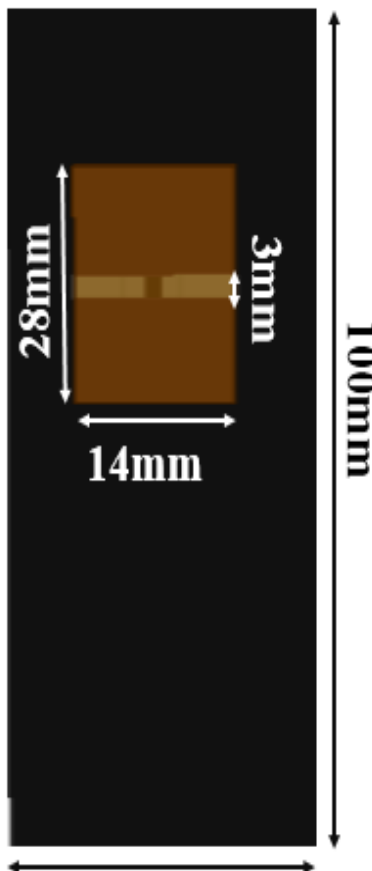

$35 \mathrm{~mm}$

(c) Schematic Model

Fig. 1. Proposed antenna

\section{RESULTS}

The performance of the antenna is measured by analyzing various parameters which includes impedance matching and radiation characteristics. Under impedance matching we will verify two parameters namely return loss also called as $S_{11}$ and Voltage standing wave ratio which is simply called as VSWR. [7]. Figure 3 and Figure 4 below shows the impedance matching characteristics of the antenna. Figure 3 depicts that the antenna is radiating at the two frequencies of $2.65 \mathrm{GHz}$ and $4.62 \mathrm{GHz}$. with return loss of $-25 \mathrm{~dB}$ and $-39 \mathrm{~dB}$ respectively. Which indicated that there is a good impedance matching for the antenna with the input power source.

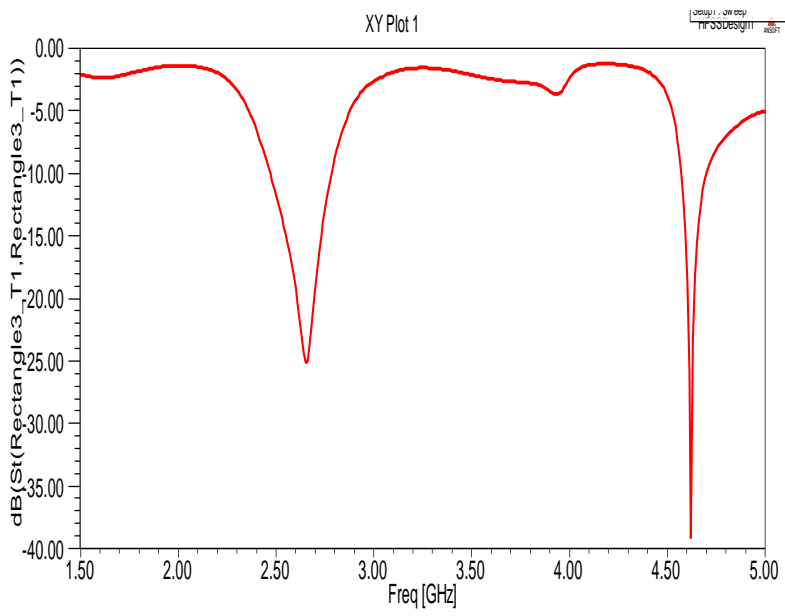

Fig. 3. Return loss

From VSWR graph in figure 4 it we can know that the antenna is radiating in the frequencies of $2.65 \mathrm{GHz}$ and $4.62 \mathrm{GHz}$ with a vswr of $1.1 \mathrm{~dB}$ and $1.2 \mathrm{~dB}$ respectively

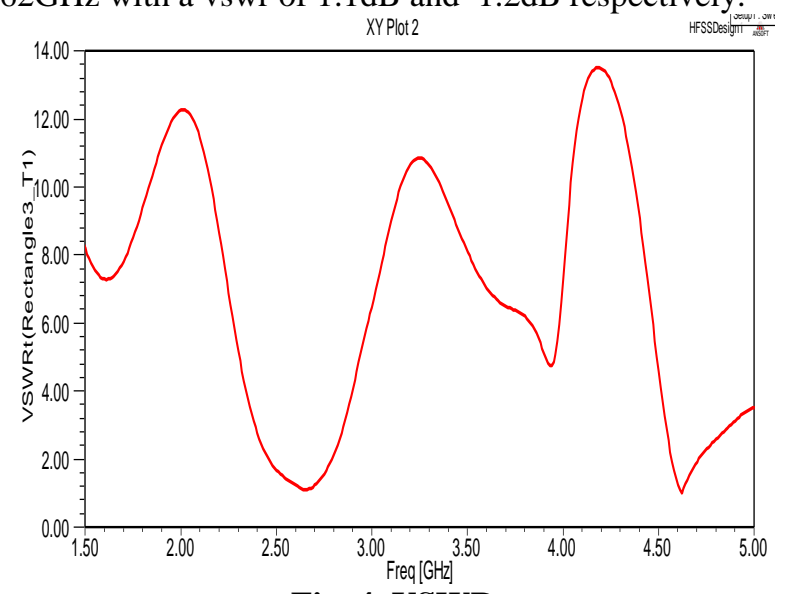

Fig. 4. VSWR

From the two gain plots in figure 5 and figure 6 below we can observe that there is a uniform distribution of the power in different theta angles without any nulls which is very essential for wireless communication applications $[8,9]$.

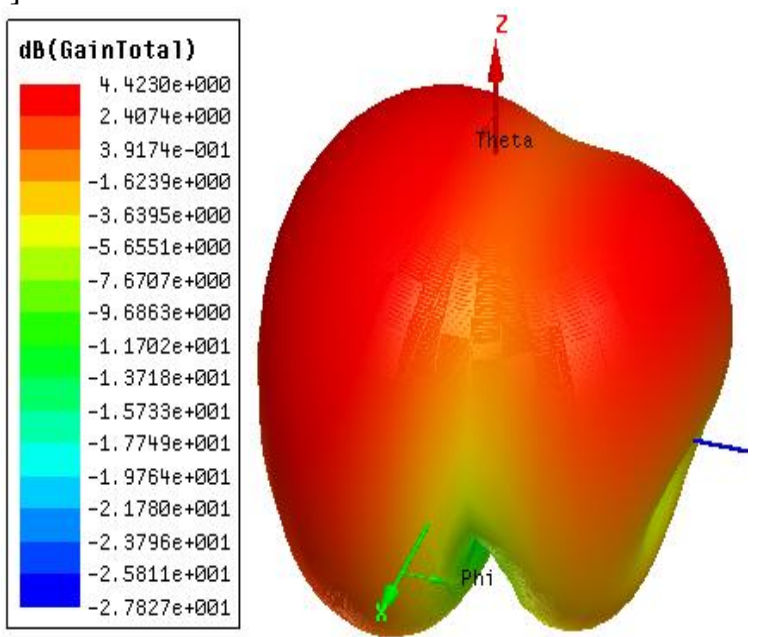

Fig.5. Gain at $2.65 \mathrm{GHz}$

Published By:

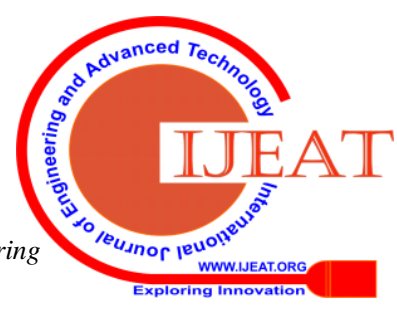



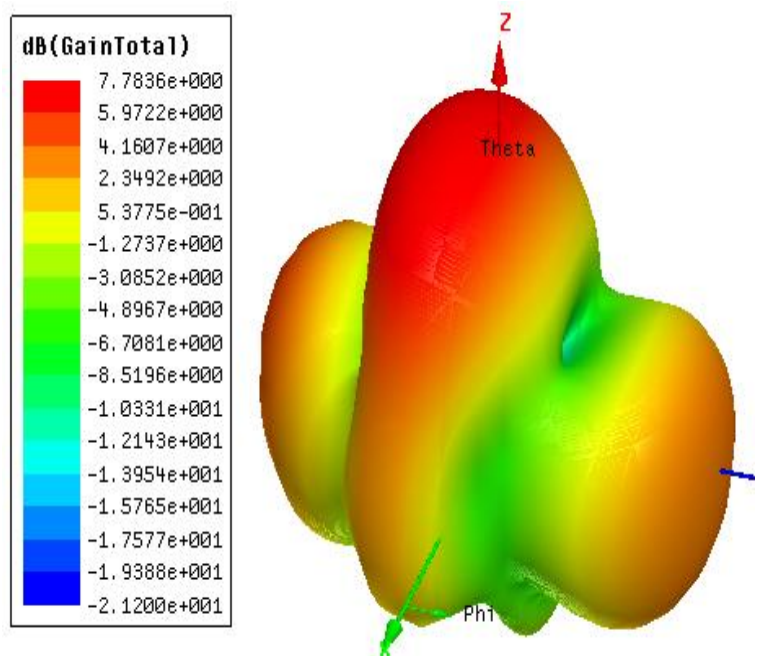

Fig. 6. Gain at 4.62GHz

The radiation characteristics of the antenna at the center frequency of $2.65 \mathrm{GHz}$ and $4.62 \mathrm{GHz}$ are shown below in Figures 7 and 8 . To analyze the radiation characteristics of the antenna we need to check both the elevation plane and azimuthal. Both the patterns are having uniform distribution of the power in different theta angles without any nulls which is very essential for the wireless communication applications for proper planning of the antenna coverage area.

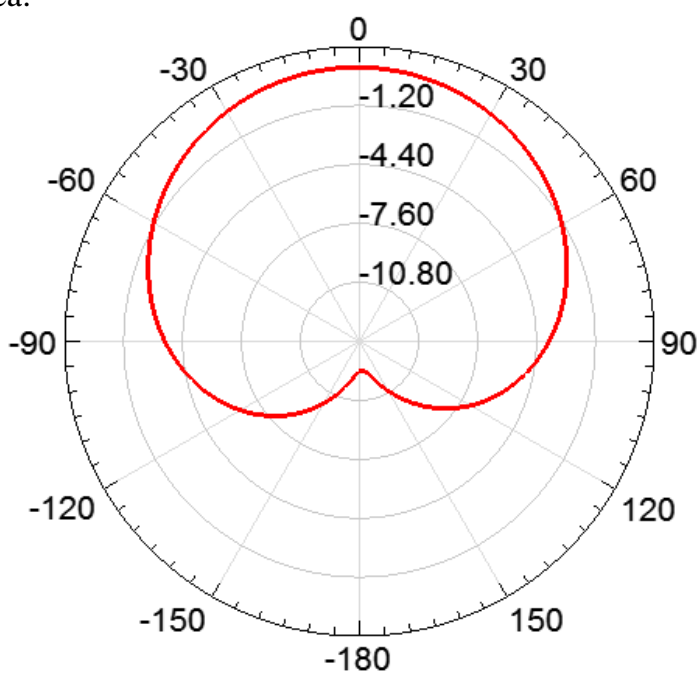

(a) Elevation Plane

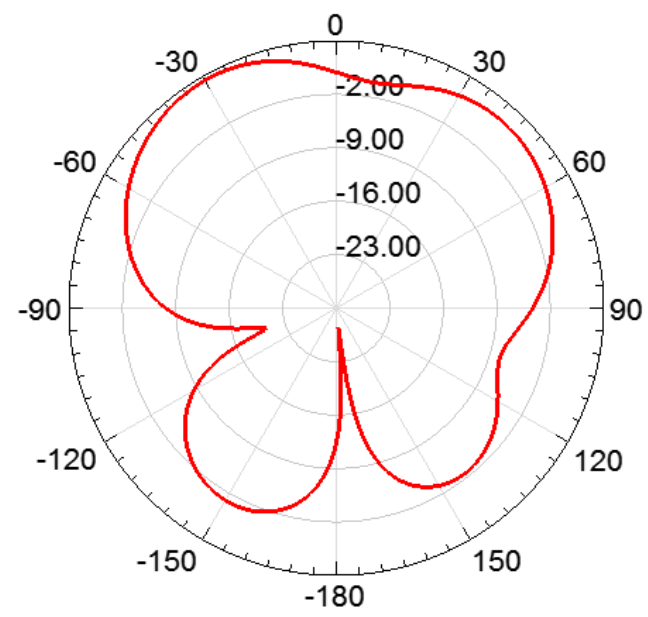

(b) Azimuthal Plane

Fig. 7. Radiation Pattern at $2.65 G \mathrm{Gz}$

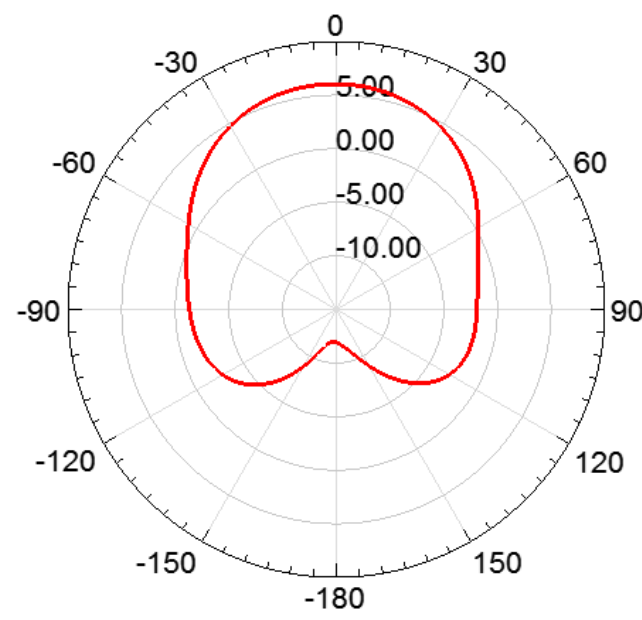

(a) Elevation Plane

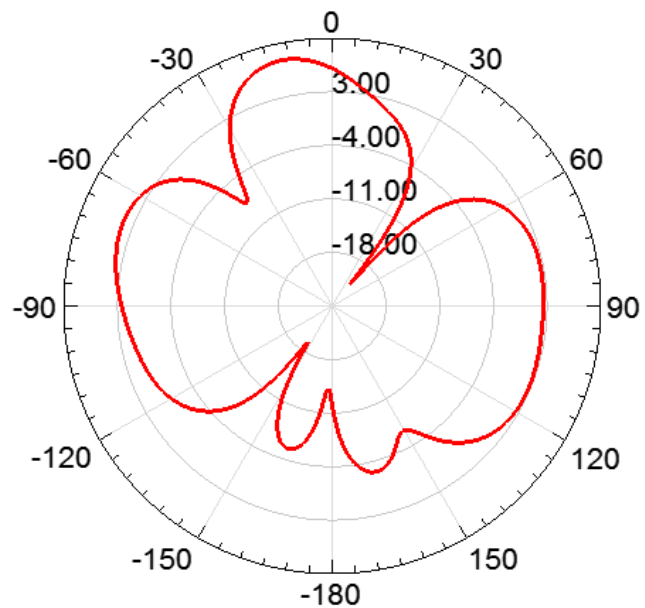

(b) Azimuthal Plane

Fig. 8. Radiation Pattern at 4.62GHz

The radiation of the antenna depends on the current fields generated in the radiating patch which are shown in the figure 11 below. We can observe that the intensity of the current field is minimum at the center of the patch and maximum at the edges. The current fields will determine the overall performance of the antenna. All the governing parameters like gain, directivity and radiation pattern of the antenna are determined from the current fields generated in the antenna. From the plots below we can observe that the current fields are different at different operating frequency of the antenna.

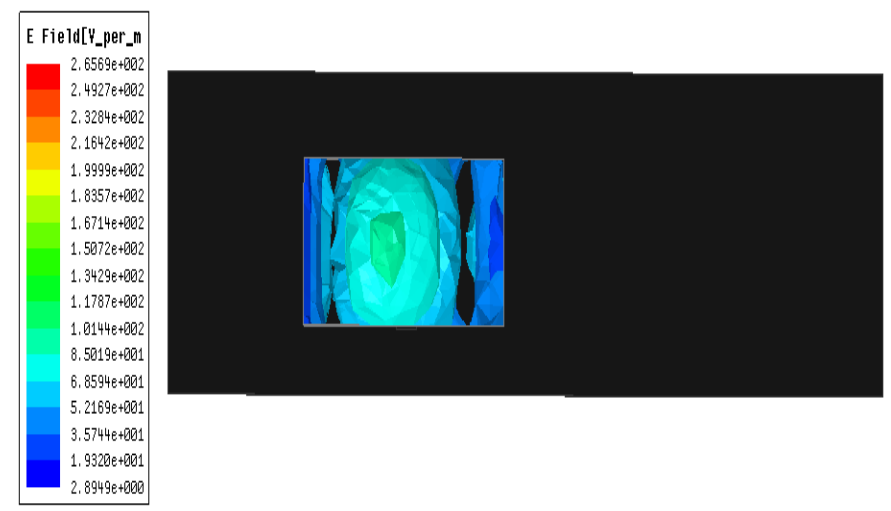

(a) lower patch at $2.65 \mathrm{GHz}$

Published By: 


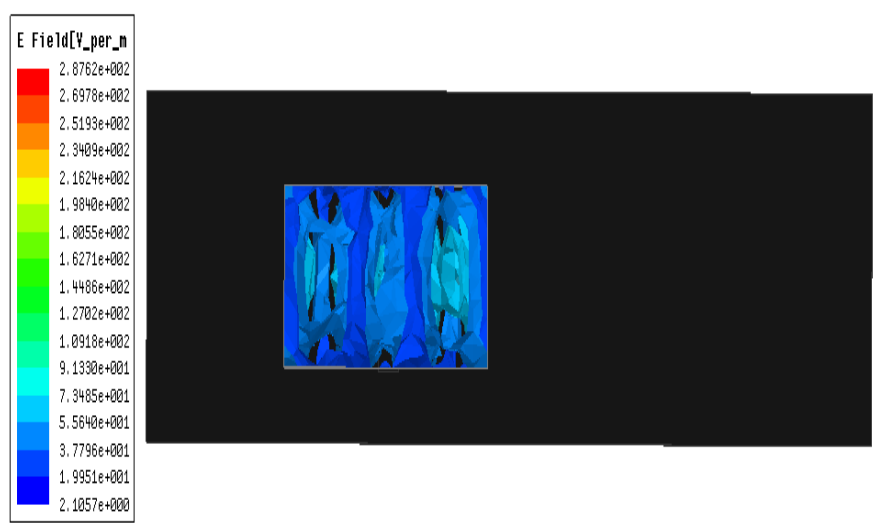

(b) Upper patch at $4.62 \mathrm{GHz}$

Fig. 9. Current distributions of the patch

\section{CONCLUSION}

A dual frequency Dielectric Resonator patch antenna has been designed for the wireless communication applications in the $\mathrm{S}$ and $\mathrm{C}$ bands with an operating frequency of $2.65 \mathrm{GHz}$ and $4.62 \mathrm{GHz}$. The patch is a dielectric material with a high dielectric constant value of 20 . A $50 \Omega$ strip line is considered as feed and is coupled to the dielectric radiator via the rectangular slot etched in ground plane. The slot etched in ground plane is made so as to facilitate the power form the feed line to the radiator. The overall dimension of the antenna is $100 \mathrm{~mm} \times 35 \mathrm{~mm} \times 0.8 \mathrm{~mm}$. A dual frequency antenna has been proposed which will be operational at the frequencies of $2.65 \mathrm{GHz}$ and $4.62 \mathrm{GHz}$ with a gain of $4.42 \mathrm{~dB}$ and $7.78 \mathrm{~dB}$ respectively. Commercially available $3 \mathrm{D}$ simulator Ansys HFSS software has been used to design the proposed antenna. The current fields will determine the overall performance of the antenna. All the governing parameters like gain, directivity and radiation pattern of the antenna are determined from the current fields generated in the antenna. From the plots below we can observe that the current fields are different at different operating frequency of the antenna.

\section{REFERENCES}

1. E. Kusuma Kumari, A.N.V.Ravi Kumar. (2017). Wideband HighGain Circularly Polarized Planar Antenna Array for L Band Radar. IEEE International Conference on Computational Intelligence And Computing Research, Tamilnadu College of Engineering. Tamil Nadu.

2. E. Kusuma Kumari, A.N.V.Ravi Kumar. (2017). Development of an L Band Beam Steering Cuboid Antenna Array. IEEE International Conference on Computational Intelligence And Computing Research, Tamilnadu College of Engineering. Tamil Nadu.

3. Sunkaraboina Sreenu, Vadde Seetharama Rao. (2017). Stacked Microstrip Antenna For Global Positioning System. IEEE International Conference on Computational Intelligence And Computing Research, Tamilnadu College of Engineering. Tamil Nadu.

4. Rao N.A, Kanapala S. (2018). Wideband Circular Polarized Binomial Antenna Array for L-Band Radar. Panda G., Satapathy S., Biswal B., Bansal R. (eds) Microelectronics, Electromagnetics and Telecommunications. Lecture Notes in Electrical Engineering, vol 521. Springer, Singapore

5. Kanapala S, Rao N.A. (2018). Beam Steering Cuboid Antenna Array for L Band RADAR. Panda G., Satapathy S., Biswal B., Bansal R. (eds) Microelectronics, Electromagnetics and Telecommunications. Lecture Notes in Electrical Engineering, vol 521. Springer, Singapore.

6. Sunkaraboina Sreenu, P. Gnanasivam, M. Sekhar (2018). Circular polarised Antenna Array for C Band Applications. Journal of Advanced Research in Dynamical \& Control Systems, Vol. 10, 14Special Issue.
7. K. Ashwini, M. Sekhar, Sunkaraboina Sreenu. (2018). Mutual Coupling Reduction Using Meander Square EBG Structures for CBand Radars. Journal of Advanced Research in Dynamical \& Control Systems, Vol. 10, 12-Special Issue.

8. Sekhar M, S Naga Kishore B, Siddaiah P. (2014). Triple Frequency Circular Patch Antenna. IEEE International Conference on Computational Intelligence And Computing Research, Park College Of Engineering And Tekhnology. Tamil Nadu.

9. J Lavanya, S Nagakishore Bhavanam, Vasujadevi Midasala "Design of Spiral Antenna for Multiband Applications” International Journal of Innovative Technology and Exploring Engineering, Volume- 8 Issue-5 March, 2019 